\title{
Impact of a Culturally Tailored Diabetes Education and Empowerment Program in a Mexican American Population Along the US/Mexico Border: A Pragmatic Study
}

\author{
Silvia Flores-Luevano ${ }^{\mathrm{a}, \mathrm{f}}$, Maricela Pacheco ${ }^{\mathrm{b}}$, Gurjeet S. Shokar ${ }^{\mathrm{c}}$, \\ Alok Kumar Dwivedi ${ }^{\text {, }}$ Navkiran K. Shokare
}

\begin{abstract}
Background: The study purpose was to deliver a diabetes education program under real world conditions and evaluate its effect on diabetes-related clinical, self-management and psychosocial outcomes among Mexican Americans residing along the US/Mexico border.
\end{abstract}

Methods: A pragmatic study was conducted among adult patients with diabetes in three primary care clinics located along the US/Mexico border. A bilingual culturally tailored diabetes education program incorporating hands-on participatory techniques was delivered in 4 - 8 weekly group sessions. Clinical, self-management and psychosocial outcomes were evaluated pre- and post-intervention with surveys and medical record review.

Results: A total of 209 participants were enrolled; mean age was 58.9 years (range 23 - 94, standard deviation: 11.2); 68.4\% were female; 91.1\% were Hispanic. Significant improvements were observed in glycated hemoglobin $(-1.1 \%, \mathrm{P}<0.001, \mathrm{n}=79)$, total cholesterol $(-17.2 \mathrm{mg} / \mathrm{dL}, \mathrm{P}=0.041, \mathrm{n}=63)$, glucose self-monitoring ( +1.3 times

Manuscript submitted June 25, 2020, accepted July 7, 2020

Published online July 22, 2020

aDepartment of Molecular and Translational Medicine and Family and Community Medicine, Texas Tech University Health Sciences Center, El Paso, TX 79924, USA

${ }^{\mathrm{b}}$ Christus Health TX A\&M College of Medicine Spohn Hospital, Family Medicine Residency, 600 Elizabeth Street, Corpus Christi, TX 78404, USA

'Department of Family and Community Medicine, Paul L. Foster School of Medicine, Texas Tech University Health Sciences Center, El Paso, TX 79924, USA

${ }^{\mathrm{d}}$ Division of Biostatistics and Epidemiology, Department of Molecular and Translational Medicine, Paul L. Foster School of Medicine, Biostatistics and Epidemiology Consulting Lab (BECL), Texas Tech University Health Sciences Center El Paso (TTUHSC EP), El Paso, TX 79905, USA

eFamily \& Community Medicine \& Department of Molecular and Translational Medicine, Center of Emphasis for Cancer, El Paso, TX 79924, USA

${ }^{\mathrm{f} C o r r e s p o n d i n g ~ A u t h o r: ~ S i l v i a ~ F l o r e s-L u e v a n o, ~ D e p a r t m e n t ~ o f ~ M o l e c u l a r ~ a n d ~}$ Translational Medicine and Family and Community Medicine, Texas Tech University Health Sciences Center, 9849 Kenworthy Street, El Paso, TX 79924, USA. Email: Silvia.Flores@ttuhsc.edu

doi: https://doi.org/10.14740/jocmr4273 a week, $\mathrm{P}=0.021, \mathrm{n}=115)$, exercise less than once a week $(-18.2 \%$, $\mathrm{P}<0.001, \mathrm{n}=129)$, nutritional behavior $(+2.23, \mathrm{P}<0.001, \mathrm{n}=115)$, knowledge $(+1.83, \mathrm{P}<0.001, \mathrm{n}=141)$ and diabetes-related emotional distress $(-7.32, \mathrm{P}=0.002, \mathrm{n}=111)$. Benefits were observed with attendance rates as low as $50 \%$.

Conclusion: A clinic-based culturally competent diabetes education/ self-management program resulted in significant improvements in outcomes among Hispanic participants. Experimentally tested culturally appropriate interventions adapted for real world situations can benefit Mexican American diabetic patients even when attendance is imperfect.

Keywords: Type 2 diabetes; Diabetes self-management education; Culturally competent interventions; Health education; Hispanics; Mexican American

\section{Introduction}

Current estimates suggest that $10 \%$ of the US population (i.e. 30 million) have diabetes including $23.8 \%$ (7.2 million) who are undiagnosed, and there are an additional 84 million with pre-diabetes [1]. This number will increase to $14 \%$ of the population by 2030 and to $18 \%$ by 2060 [2]. There is a greater diabetes prevalence among racial/ethnic minority groups; among US Hispanics, the prevalence is $12 \%$ and among the Mexican American subgroup, it is nearly 14\% [1]. Diabetes is associated with excess medical costs: in 2012, the total direct medical expenses for all type of diabetes in the US were estimated at $\$ 18.2$ billion and indirect costs were $\$ 5.5$ billion [3]. Diabetes is the seventh leading cause of death in the US with an average 4.4 years of life lost per person [4]. Mortality rates among Hispanics are higher than both national and state rates $(24.7 / 100,000$ vs. $21.5 / 100,000)$ [5], and may be even higher because diabetes is an underreported cause of death [6]. Diabetes especially impacts older adults, racial/ethnic minorities, men and those with lower educational attainment. Progressively, diabetes may lead to blindness, kidney failure, myocardial infarction, stroke, painful neuropathy, foot ulcers and lower limb amputation [7]. Previous work has shown that Hispanics 
are at greater risk of uncontrolled diabetes, diabetic complications and death from diabetes than non- Hispanic whites [8$10]$.

The Association of Diabetes Care \& Education Specialists (ADCES) identifies seven self-care behaviors that predict good outcomes in people with diabetes including blood sugar monitoring, compliance with medications, good problem-solving skills, healthy coping skills and risk-reduction behaviors [11]. Hispanics are particularly prone to have poor self-management skills in adherence to ADCES's seven essential behaviors, specifically in monitoring their glycated hemoglobin (A1c) [12]. Data suggest that culturally competent self-management educational programs, along with medical management implemented in controlled experimental designs can improve clinical outcomes through reduction in A1c, improved patient knowledge and dietary behaviors [13-18]. Several systematic reviews conclude that diabetes self-management education (DSME) programs in adults employing different delivery methods have been effective in diverse settings among general populations [19, 20] including Hispanics [21].

The data summarized above reveal that under experimental conditions education and self-management programs can improve important diabetes outcomes; however, despite this evidence, diabetes-associated morbidity and mortality remain high particularly in Hispanics. This suggests that these intervention types are either not being implemented in practice or do not achieve similar outcomes in the real world. Relatively few studies with limited behavioral and psychological outcomes have yielded the promising effect of a diabetes education intervention program in the US population. Moreover, no previous studies have determined the effectiveness of a diabetes education and self-management program in a pragmatic and uncontrolled setting particularly in Mexican Americans who have higher incidence of diabetes and associated complications. Compared to prior studies, our study also proposes to determine the effect of this intervention on behavioral and psychological parameters. The aim of this study therefore was to implement and evaluate a culturally tailored diabetes education and self-management program in a clinic setting under real world conditions. The novelty of this study is to report that even in a non-experimental control intervention, diabetes practical education can be implemented and adapted to patient's needs. In addition, we included a range of outcome variables for which there is limited information available and we described implementation challenges and proposed solutions.

\section{Materials and Methods}

\section{Study design and setting}

This was a pragmatic non-randomized diabetes education and self-management support intervention that utilized a pre-post design for evaluation of outcomes. Participants were recruited from three primary care clinics located in El Paso, Fabens and Socorro Texas along the US-Mexico border. Over $80 \%$ of the population in these regions is Hispanic and between $21 \%$ and $29 \%$ live below the poverty level [22]. All program staff were bilingual, and the study was approved by Institutional Review Board of Texas Tech University Health Sciences Center, El Paso (IRB Protocol \# E11081).

\section{Eligibility}

Individuals were eligible if they were a patient in one of the participating clinics, were aged 18 years or older and had a documented diagnosis of diabetes. Patients were either referred by their primary care physician, self-referred through word of mouth, or through flyers posted in the clinics. Potential participants were contacted by staff to check eligibility, and explain the study between June 2011 and April 2014. Exclusion criteria included inability to provide informed consent or a cognitive, mental health, pregnant women, or a medical condition such as cancer that could impact participation. An informed consent and HIPAA consent were obtained from the patients for the study.

\section{Intervention}

The Diabetes Education and Empowerment Program (DEEP) curriculum is a multi-cultural and bilingual (English and Spanish) eight module program that was created by the University of Illinois [23]. It is tailored for Hispanic (as well as African American) populations to improve diabetes self-management through education about diabetes, risk factors and complications, diet, physical activity, and use of a glucometer and medications. The DEEP curriculum provides resources for patient empowerment through partnerships with a diabetes health care team, promoting understanding of the psychosocial effects of illness, problem-solving strategies, and guiding patients in how to access community diabetes resources. The curriculum is sensitive to cultural issues and popular misconceptions about diabetes in the Hispanic community. The sessions were interactive with demonstrations, activities to promote problem-solving, and facilitated group dynamics through the sharing of personal experiences.

\section{Procedure}

After written informed consent was obtained, participants completed a pre-intervention questionnaire which assessed clinical demographic and behavioral information. The 2-h classes were delivered weekly over 4 - 8 weeks. Initially eight weekly sessions were provided but these were integrated into six and then four weekly sessions to reduce attrition. Separate classes were available for English and Spanish speaking participants. The curriculum was implemented by clinic promotoras (community health workers), a health educator and a research assistant who received training on program delivery. The post-intervention survey was administered after the final session. Completion of the surveys took a maximum of $1 \mathrm{~h}$. The clinical outcomes were abstracted from the electronic medical record (EMR) using a standardized protocol for the 
period of $6-12$ months prior to the intervention and $6-12$ months after the last session for each participant.

\section{Measures}

Survey variables covered socio-demographic information (gender, race/ethnicity, health care coverage, education level, country of birth, income and preferred language). Acculturation was assessed with the validated short acculturation scale for Hispanics [24] which is a four-item scale that is scored on a five-point Likert-scale with one being only Spanish to five being only English.

Diabetes history questions covered duration of diagnosis, diabetes type, previous diabetes education, A1c awareness, doctor visits and receipt of foot and eye exams. Diabetes knowledge was assessed with a modified Starr County Diabetes Knowledge Questionnaire consisting of 21 true-false questions (Cronbach's alpha coefficient $=0.7$ ) [25]. Responses were coded as correct or incorrect and the score was summed for each participant. Three self-management behaviors were evaluated: eating behaviors were evaluated with the Latino Dietary Behaviors Questionnaire (LDBQ), a survey developed for the Spanish-speaking community with 13 questions answered on a scale of 0 to 4 or 0 to 5 . A total score and factor scores are calculated by summing across individual items. A higher score reflects healthier eating behaviors with an internal reliability of 0.47 and test-retest reliability of 0.48 [26]. Physical activity was measured by items assessing hours per day watching TV/videos and times per week exercising. Glucose monitoring frequency was assessed with one item. Two psychosocial variables were assessed: diabetes related emotional distress was measured with the problem areas in diabetes (PAID) scale which consists of 20 questions with a Cronbach's alpha of 0.90 , and test-retest reliability of 0.83 [27]. The Likert scale is scored between 0 and 4, from "not a problem" to "a serious problem" then the answers are totaled and multiplied by 1.25 . Total scores of 40 and above suggest severe diabetes distress (Supplementary Material 1, www.jocmr.org). Fatalism was assessed with the diabetes fatalism scale, a 12item survey with three sub-scales reflecting emotional distress, religious, spirituality coping and perceived self-efficacy. The scale is scored from 1 to 6 on a Likert scale, has an internal reliability of 0.80 and correlates well with A1c [28]. Clinical outcomes (A1c, lipid levels, blood pressure and body mass index (BMI), retinal eye exam and foot exam) were assessed by EMR review for each participant at 6 - 12 months pre-intervention and at 6 - 12 months post-intervention.

\section{Data analysis}

Descriptive analyses described survey and clinical diabetes measures. Quantitative variables were reported as the mean and standard deviation (SD) while categorical variables were reported with frequency and percentage. For knowledge and behavioral scales (fatalism, LDBQ and PAID), we calculated the change in scores from baseline to post-education using mean percentage change along with $95 \%$ confidence interval (CI). The effect of the intervention was also reported by calculating the change in scores from pre- and post-intervention in individuals using paired $t$-tests. Changes in pre- and post-intervention clinical outcomes of A1c levels, BMI, cholesterol, high-density lipoprotein-cholesterol (HDL-C), low-density lipoprotein-cholesterol (LDL-C), triglyceride (TG) and urinary microalbumin were calculated and analyzed by paired $t$-test. Predictors of glycemic control defined as A $1 \mathrm{c} \leq 8 \%$ at follow-up were examined using logistic regression analysis. Results of logistic regression were reported using odds ratio (OR) with 95\% CI and P-value. In the sensitivity analysis, the psychosocial, diabetes knowledge and clinical outcomes between pre- and post-intervention were compared using a paired $t$-test by educational session attendance percentage as $<50 \%$ of attendance, between $50-74 \%$ and $\geq$ $75 \%$. SPSS software version 25.0 was used for data analysis and an alpha of 0.05 was used to determine statistical significance. Important results were summarized using forest plots.

\section{Results}

Of 275 potential participants contacted, 209 were eligible and enrolled in the study. The intervention was conducted in 13 education cycles from June 2011 to April 2014. A total of 143 females and 66 males participated. Attrition was observed for class attendance: $31.6 \%(n=66)$ of participants attended all the sessions, $58.9 \%(n=123)$ attended $75 \%$ or more of the sessions and $76.1 \%(n=159)$ attended at least $50 \%$ of the sessions (Fig. 1). Mean age was 58.9 years; $91.1 \%$ were Hispanic; $74.3 \%$ reported an annual income less than $\$ 20,000$, about half had a high school diploma $(57.1 \%)$ and $27 \%$ were uninsured. Over half of the participants $(55.9 \%)$ were born in Mexico and $58.7 \%$ reported a Spanish language preference and had low acculturation levels $(72.3 \%)$. The majority of participants rated their health as fair or poor (58\%). Almost half of participants reported watching TV/videos more than $2 \mathrm{~h}$ per day $(51.2 \%)$ and the majority $(73 \%)$ reported participation in sports or exercise less than one time per week.

The mean duration of diabetes was 8 years (SD: 7.8), $20 \%$ did not know their diabetes type and most participants $(61.5 \%)$ had never attended a diabetes class. The majority $(82.9 \%)$ reported monitoring their glucose at least once per week and sought diabetes care at least once per year $(91.9 \%)$. Sixty-eight percent reported a doctor checked their feet in the previous year and $98.8 \%$ had completed at least one eye exam. Only $28.6 \%$ reported knowing their A1c test result and 26.9\% reported retinopathy. The clinical and demographic characteristics of the study participants are described in Tables 1 and 2 . There were no significant differences in the baseline sociodemographic characteristics of completers $(n=123)$ (i.e. completed $75 \%$ of sessions) and non-completers $(n=86$, Table 1$)$. A relatively lower frequency of foot examination by a doctor was reported among non-completers (Table 2).

\section{Clinical outcomes}

Among all participants, the mean baseline A1c was $8.98 \%$ 


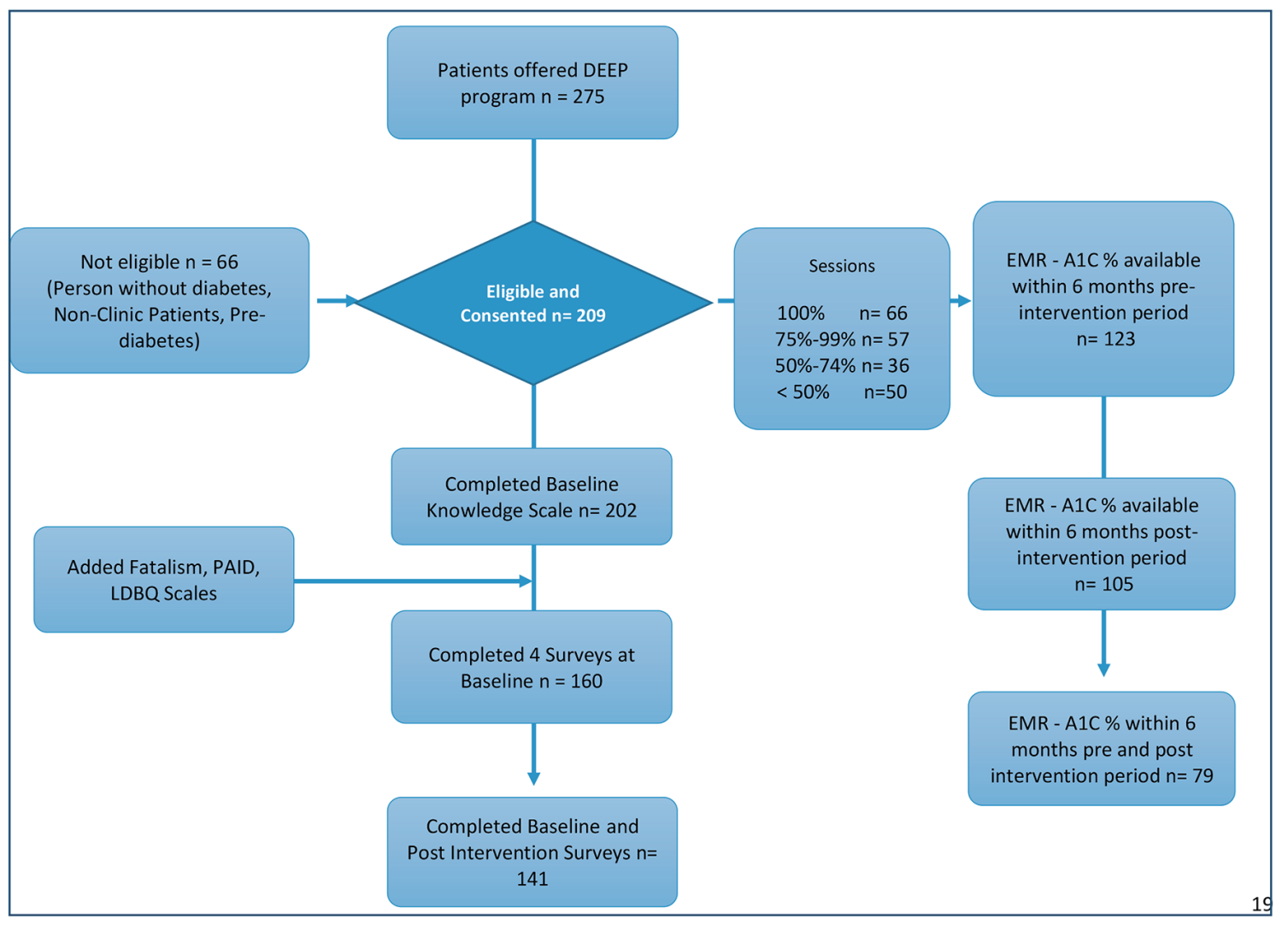

Figure 1. Flowchart of the study participants. DEEP: Diabetes Education and Empowerment Program; EMR: electronic medical record; PAID: problem areas in diabetes; LDBQ: Latino Dietary Behaviors Questionnaire.

(211 mg/dL) (SD: 2.37), the BMI was 32.68 with $60.5 \%$ in the obese BMI range. Mean total cholesterol was $186.55 \mathrm{mg} /$ dL (SD: 55.81 ) and $31.9 \%$ had a total cholesterol level $>200$ $\mathrm{mg} / \mathrm{dL}$; mean LDL-C was $98.31 \mathrm{mg} / \mathrm{dL}$ (SD: 37.58 ), $50 \%$ of participants had an LDL-C level $<100 \mathrm{mg} / \mathrm{dL}, 26.9 \%$ were in the 100 to $129 \mathrm{mg} / \mathrm{dL}$ range, $11.1 \%$ were in the 130 to 159 $\mathrm{mg} / \mathrm{dL}$ range and $8.3 \%$ had LDL-c level $\geq 160 \mathrm{mg} / \mathrm{dL}$; mean HDL-c was $43.66 \mathrm{mg} / \mathrm{dL}$ (13.69) and mean TGs were 172.17 $\mathrm{mg} / \mathrm{dL}$ (86.57). Among the 40 participants with an available microalbumin level, the mean was $77.81 \mathrm{mg} / \mathrm{dL}$ (SD: 156.33), and $47.5 \%$ had a microalbumin $>30 \mathrm{mg} / \mathrm{dL}$ (data not shown).

Table 3 shows the results of the paired analysis among participants that had both pre- and post-intervention clinical variables available in the medical record and behavioral and psychological variables collected.

\section{Clinical outcomes pre-post intervention}

The A1c declined significantly from $8.92 \%$ to $7.82 \%(-1.10 \%$, $\mathrm{P}<0.001, \mathrm{n}=79)$, and $67 \%(\mathrm{n}=52)$ of participants had a reduction in A1c from pre- to post-intervention. The proportion of participants with an A1c level $>8 \%$ (i.e. uncontrolled) also declined significantly (from $56 \%$ to $39 \%, \mathrm{P}<0.001$ ). The total mean cholesterol level declined from $188 \mathrm{mg} / \mathrm{dL}$ (SD: 60) to $171 \mathrm{mg} / \mathrm{dL}$ (SD: 37$)(\mathrm{P}=0.04, \mathrm{n}=63)$ at 6 months post- intervention. Although TG, HDL, LDL and urinary microalbumin levels improved compared to their respective baseline values, the changes did not reach statistical significance and no changes were observed in the BMI post-intervention.

\section{Diabetes self-management behaviors pre-post intervention}

Significant improvement was also observed in all three diabetes self-management behaviors: glucose self-monitoring increased by $16 \%$ from pre- to post-intervention (8.21 times per week vs. 9.54, $\mathrm{P}=0.021, \mathrm{n}=115)$; with lower numbers of participants reporting never checking their glucose $(32 \%$ vs. $17 \%$ ). A greater percentage reported exercising more than three times per week ( $14 \%$ vs. $28 \%$ post) and exercising 1 to 3 times per week ( $13 \%$ vs. 17\%), whereas those reporting exercising less than once per week declined ( $73 \%$ vs. $55 \%$ post) $(\mathrm{P}=0.001, \mathrm{n}=129)$. Self-reported beneficial dietary behaviors measured using the LDBQ also showed a significant increase (mean change $=2.23, \mathrm{P} \leq 0.001, \mathrm{n}=115$ ) from pre- to postintervention (Table 3).

\section{Knowledge, diabetes emotional distress and fatalism}

The results related to knowledge, diabetes emotional distress 
Table 1. Baseline Characteristics of Participants

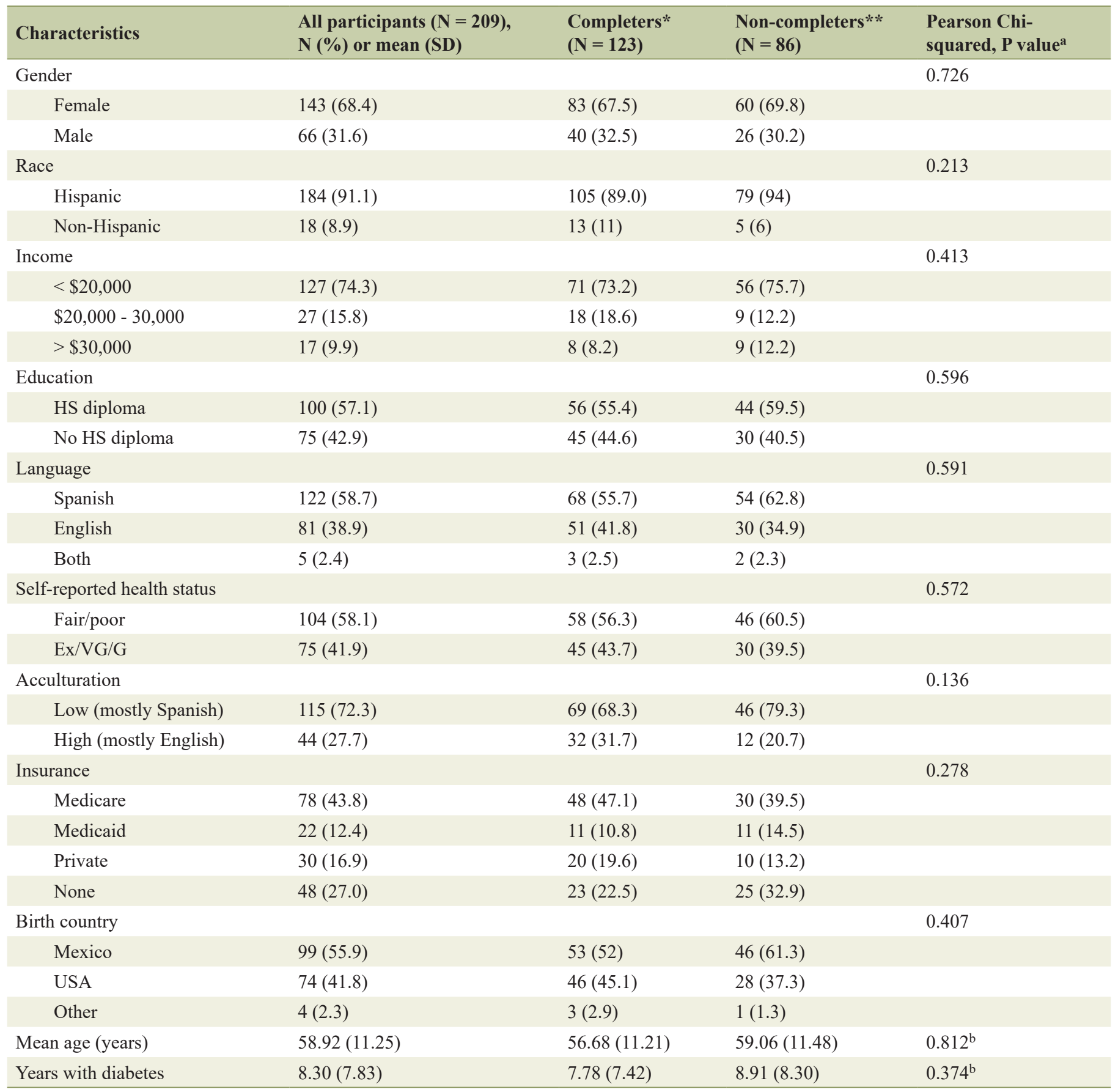

${ }^{*}$ Attended $\geq 75 .{ }^{*}$ Attended $<75$. ${ }^{a}$ Completers vs. non-completers. ${ }^{\mathrm{b}} T$-test.

and fatalism are reported in Table 3 . The mean baseline diabetes knowledge score was 13.45 (SD: 3.33, maximum possible score: 21). Lack of knowledge was especially observed for detecting symptoms for variation of blood glucose: "shaking and sweating are signs of high blood sugar" (67.3\% incorrect) and "frequent urination and thirst are signs of low blood sugars" (54.9\% incorrect). The mean PAID scale score was 39.22 (SD: 26.66) and half of participants had a significant diabetes emotional distress level (i.e., a score $\geq 40$ ). All the psychosocial outcomes improved significantly after the intervention; mean knowledge scores increased 1.83, $(\mathrm{P}<0.001, \mathrm{n}=141)$; PAID scores decreased by a mean of -7.32 , and the proportion with significant diabetes distress (PAID scores $\geq 40$ ) decreased from $51.4 \%$ to $38.7 \%(\mathrm{P}<0.001, \mathrm{n}=111)$. Fatalism also decreased but the change was not statistically significant $(-1.22$, $\mathrm{P}=0.39, \mathrm{n}=110)$. The maximum effect size observed across 


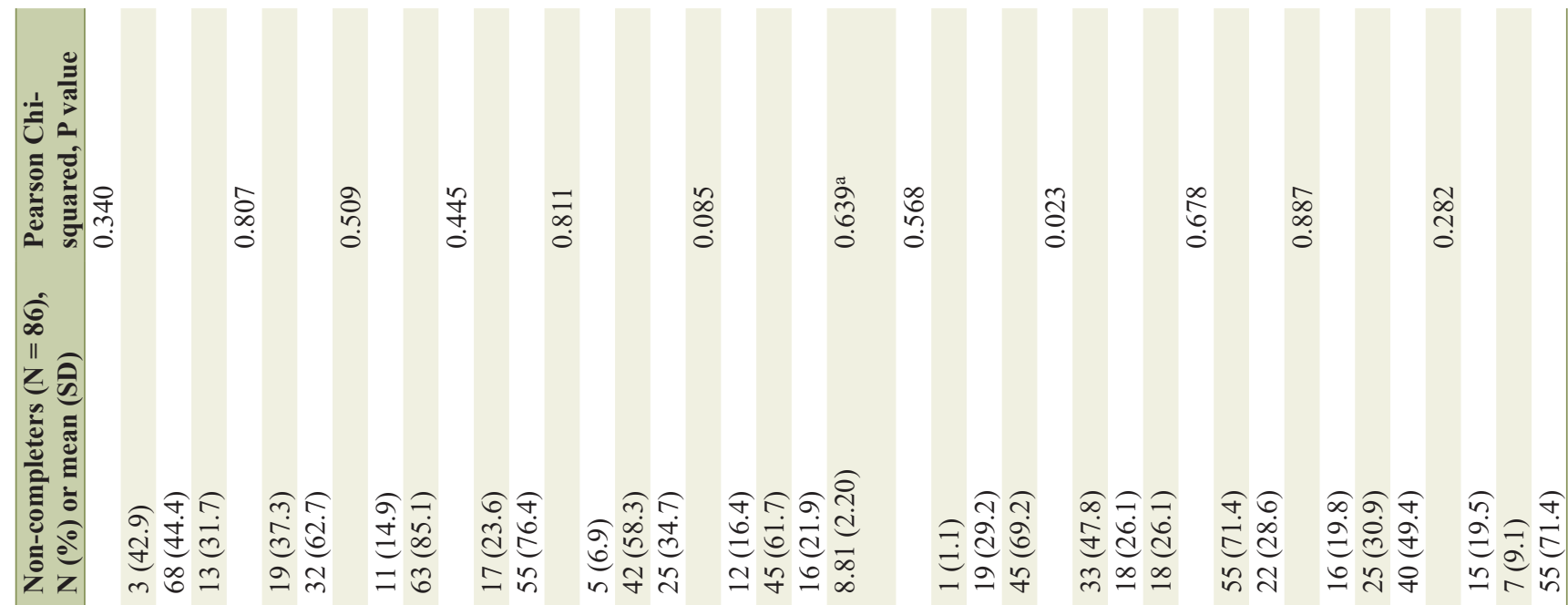

กิธิซี

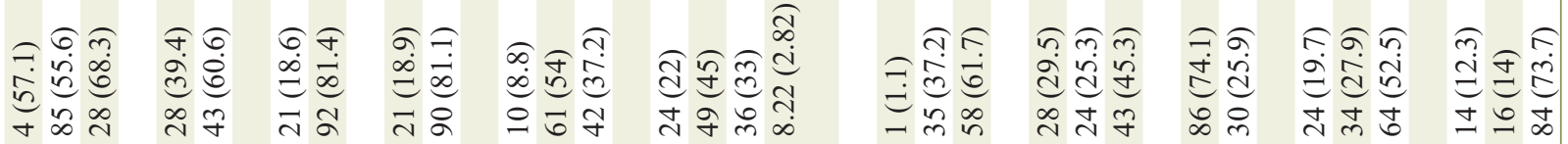

ิิ

乙艺密

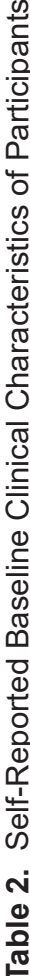

产

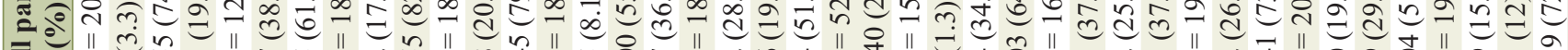

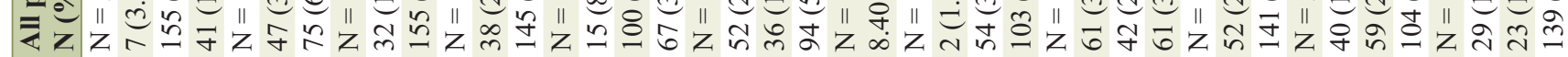

$\stackrel{\frac{0}{0}}{\frac{0}{6}}$
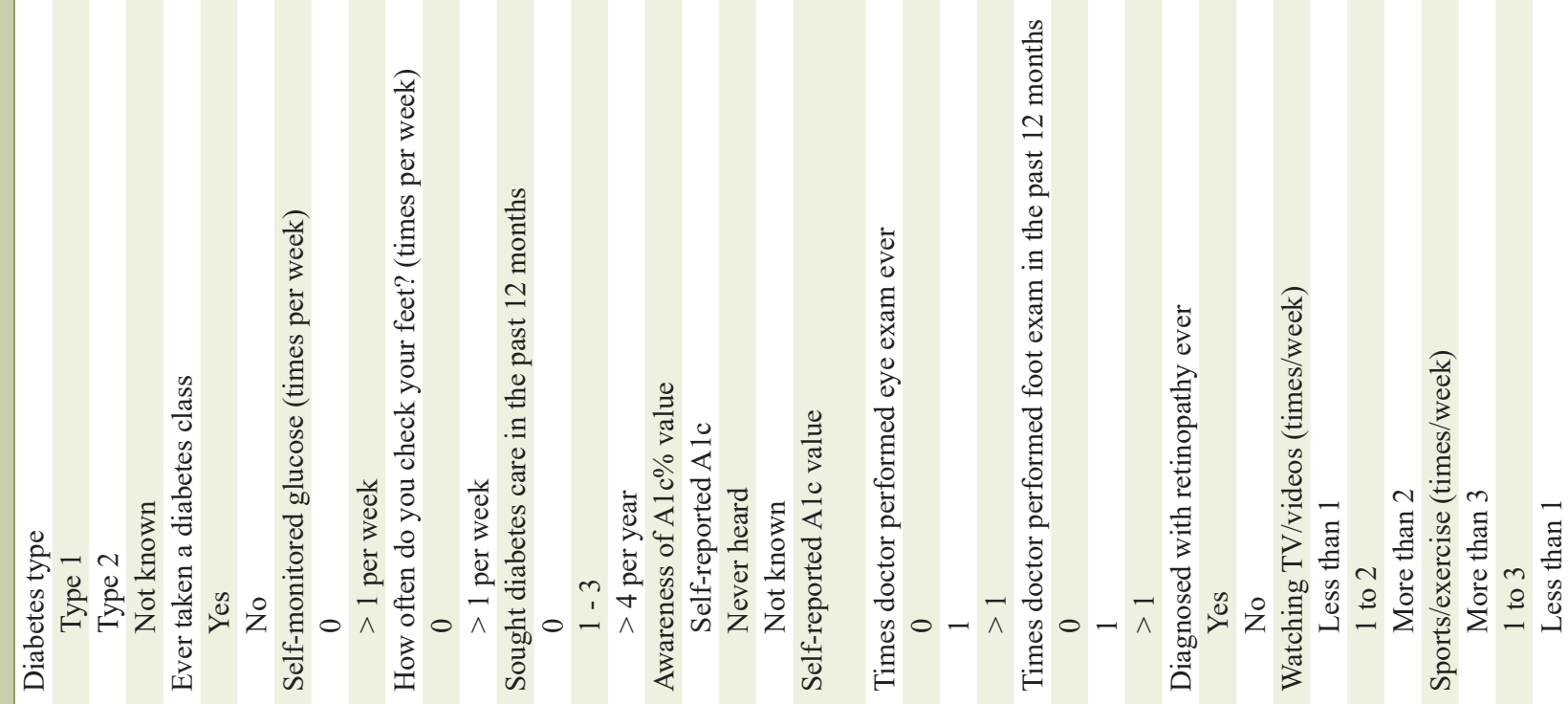


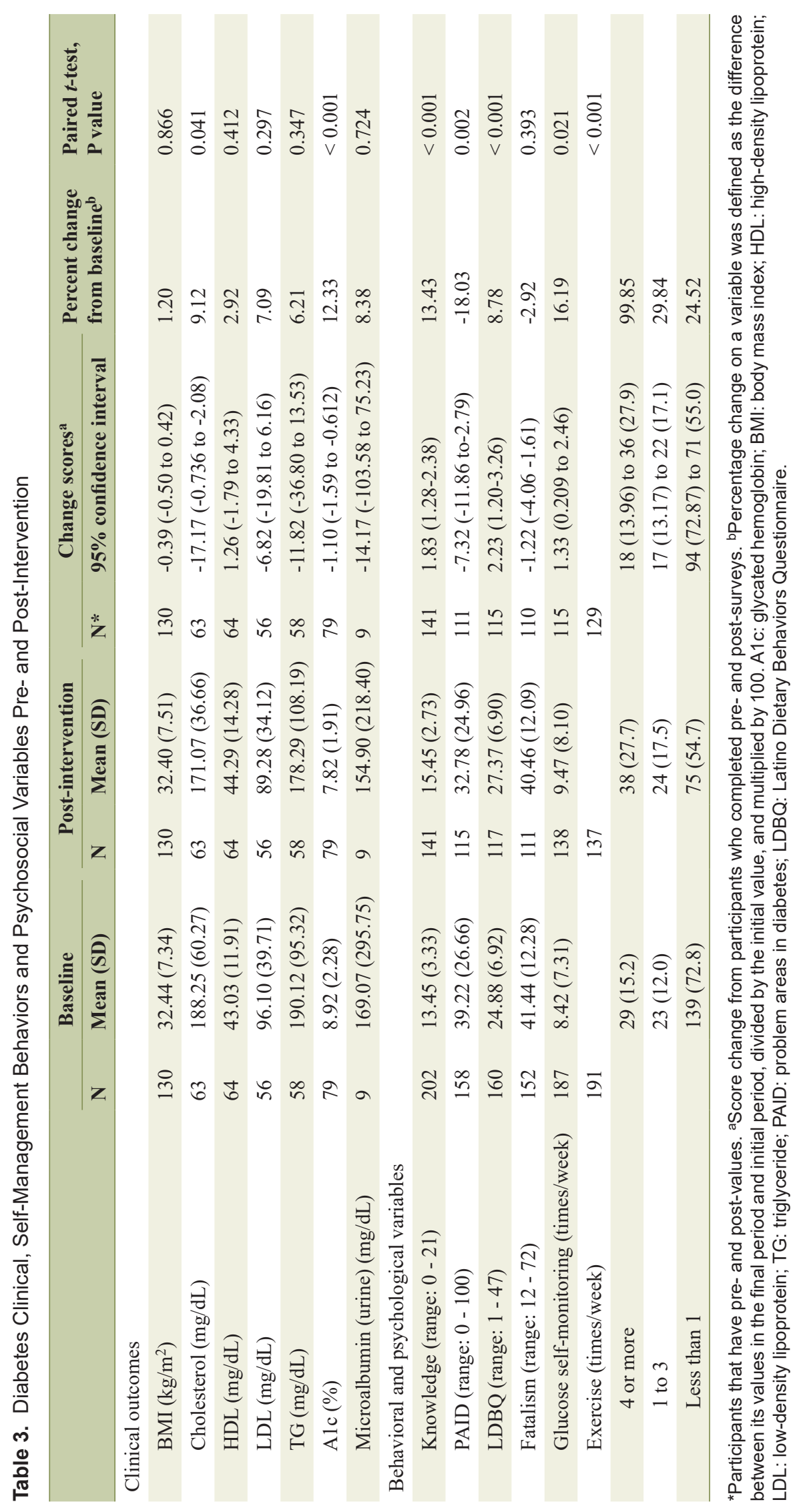


Table 4. Unadjusted Associations Between Demographic and Behavioral Characteristics With Glycemic Control (A1c $\leq 8 \%)$

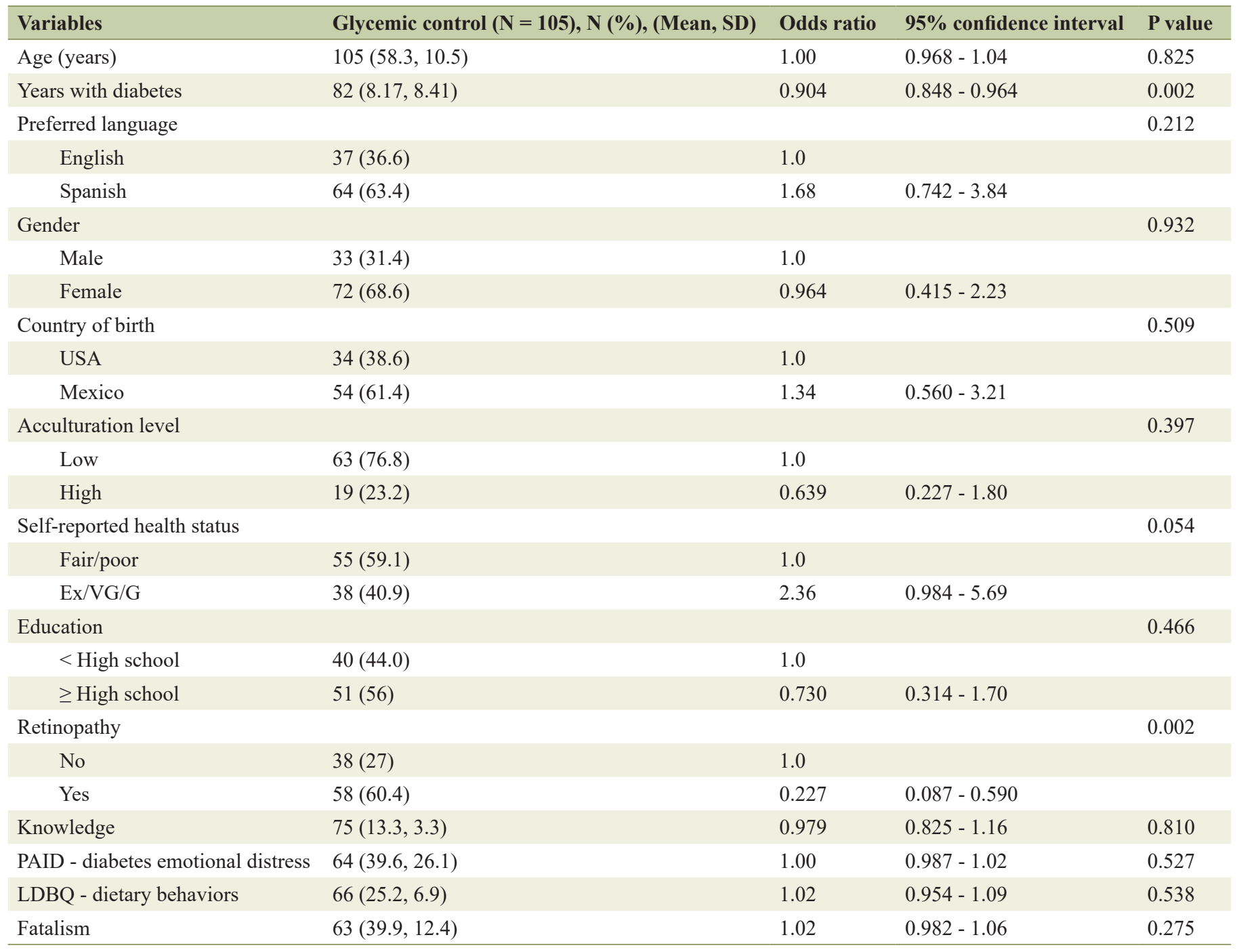

PAID: problem areas in diabetes; LDBQ: Latino Dietary Behaviors Questionnaire.

psychosocial variables was for diabetes emotional distress (18\%) followed by knowledge (13\%), and then fatalism (3\%).

\section{Predictors of glycemic control}

In the logistic regression analysis, we examined the association between baseline demographic characteristics, clinical and psychosocial variables and post-intervention glycemic control $(\mathrm{A} 1 \mathrm{c} \leq 8)$ as an outcome variable. The A1c value was available and abstracted from the EMR during a period of 6 12 months of follow-up for 105 participants. A greater number of years of diagnosed diabetes and diagnosed retinopathy were both associated with lower odds of glycemic control $(\mathrm{OR}=$ $0.904, \mathrm{P}=0.002$ and $\mathrm{OR}=0.227, \mathrm{P}=0.002$, respectively). Better health status (good, very good or excellent vs. poor or fair) was associated with a greater odds of glycemic control $(\mathrm{OR}=$ $2.36, \mathrm{P}=0.054)$. Diabetes knowledge, psychosocial and self- management variables were not significantly associated with glycemic control in this analysis (Table 4).

Effect of education session attendance on clinical, knowledge, psychosocial and diabetes self-management outcomes

Because of the relatively high attrition rate, a final analysis of outcomes was performed stratified by attendance. The majority of the study participants who completed the intervention had $75 \%$ or more sessions completed. Although not statistically significant, the effect size for most outcomes was markedly higher in patients who completed $50-74 \%$ of sessions compared to those who did not $(<50 \%)$ with similar sample sizes. Moreover, A1c, knowledge and the PAID score showed improvement even for those attending a moderate level of sessions (50-74\%). Those who did not complete sessions had an opposite effect size compared to those who attended a mod- 


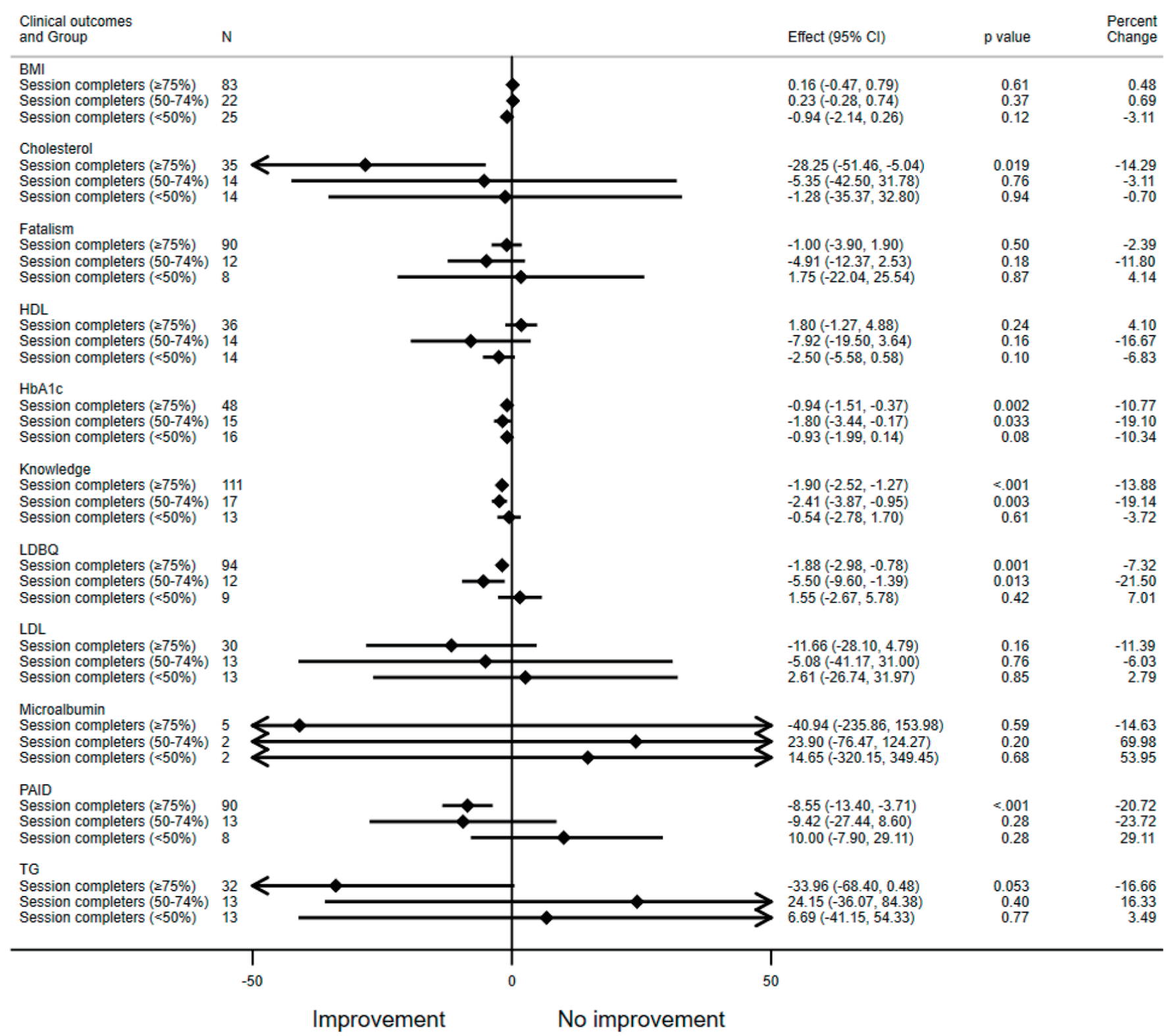

Figure 2. Effect of intervention by sessions attendance. All variables are presented with pre-post changes except for HDL, knowledge and LDBQ (post-pre changes). HDL: high-density lipoprotein; LDBQ: Latino Dietary Behaviors Questionnaire.

erate level of sessions. Completers demonstrated significant improvement in the majority of clinical and behavioral parameters (Fig. 2).

\section{Discussion}

We implemented a culturally tailored and literacy level-appropriate diabetes education program in a predominantly Mexican American population. Significant findings of this study include improvements in clinical (glycemia, cholesterol), self-management (glucose self-monitoring, exercise and diet), knowledge and psychosocial outcomes. A key finding of this study was an improvement in glycemia 6 months after the intervention: the proportion of participants having glycemic control (A1c $\leq$ 8 ) increased after the intervention from $43.9 \%$ to $60 \%$ and the mean A1c level decreased from $8.92 \%$ to $7.82 \%$. This change is nearly twice the $0.6 \%$ reduction published in a meta-analysis of culturally appropriate diabetes education interventions among minorities that included 11 studies from across the world and 1,603 patients [29] and is higher than those reported in a systemic review of 18 DSME interventions that included 3,540 adult Latinos with type 2 diabetes where the pooled effect on A1c was a reduction of $0.24 \%$ [21]. A change in A1c level from $8.92 \%$ to $7.82 \%$ indicates that the typical study participant dropped from an average daily blood glucose of 207 
to $177 \mathrm{mg} / \mathrm{dL}$, a noticeable change in daily self-management [30].

We observed a significant reduction of total cholesterol level $(-17.17 \mathrm{mg} / \mathrm{dL}, \mathrm{P}=0.041)$ at 6 months post-intervention which is similar to the changes observed in a randomized intervention of a 3-month self-management education among Mexican Americans with diabetes living along the TexasMexico border (-21.95) [31] and much higher than the changes observed by others [29].

We observed non-significant improvements in LDL-C, HDL-C and TG.

We also observed improvements in all three considered diabetes self-management behaviors (glucose self-monitoring, healthy eating and physical activity). Self-care practices depend on access to education, health status, self-efficacy, social and family support, cultural factors and the relationship with primary health professionals $[32,33]$. Even though Hispanic Americans may be more interested than non-Hispanic whites in improving self-management behaviors [34], challenges for self-management in Hispanics include barriers to healthcare access, transportation, lack of quality health care and costs (e.g. checking blood glucose less times that recommended to reduce costs) [35]. Glucose self-monitoring among adults with diabetes is an effective tool for achieving glycemic control and for reducing chronic diabetes complications. It has been reported to raise awareness of this chronic disease, to prevent major health problems and help to interpret signs and symptoms of diabetes [36], even though, self-monitoring is difficult to sustain over time [32]. We also observed significant changes in physical activity levels: at baseline $72 \%$ of participants reported being physically inactive (less than one time per week of doing exercise) and this was reduced to $54.7 \%$ after the intervention. However, despite the improvement, this percentage remains higher than the $41 \%$ reported in a national sample of diabetics who reported being inactive [1].

Participants demonstrated statistically significant improvements in knowledge and diabetes-related emotional distress (PAID). Similar improvements in diabetes knowledge have been noted previously $[31,37]$. Participants perceived a higher emotional distress at baseline which was significantly reduced after the intervention. These results are consistent with the findings observed in diverse clinical settings [38], and for patients undergoing one-on-one interventions [39]. This result has potential positive implications because of the reported relationship between higher diabetes distress and poor selfmanagement, worse medication adherence, depression and poor quality of life [40, 41].

There are some interesting points to note about this population at pre-intervention. Although the average duration of diabetes was 8 years, almost $20 \%$ reported not knowing which type of diabetes they had, and 70\% had not heard about A1c or were aware of its purpose despite having an average of over three doctor visits per year. This may be because only $38.5 \%$ reported attending a diabetes class which is a lower percentage than the approximately 50\% reported nationally [37] and in Texas in 2017 [42]. However, they did report high levels of positive behaviors both by themselves or their physicians which may suggest potential over-reporting.

Of note, the main predictors of less glycemic control were having more years with diabetes and retinopathy which are the consequences of chronic hyperglycemia in this population [43].

Despite proven benefits, there are challenges in implementing and evaluating evidence-based interventions in real world practice. We encountered many challenges which required a number of adaptations to address them. First we had to schedule separate sessions in English and Spanish; second we dropped the smoking module because of a low rate of community smoking rates; third we reorganized the sessions into 6-week and then 4-week sessions in order to increase adherence from week to week; fourth we added multiple phone reminders and permitted participants to attend missed sessions in other classes in order to improve follow-up. We had additional challenges in extracting the clinical information because our EMR changed during study implementation, requiring us to access two separate EMR systems. Finally, we examined program outcomes based on attendance, and this clearly showed that there is continuous improvement in outcomes with number of sessions attended. So even partial completion of the intervention had some positive impacts on outcomes. This is useful information for program planners and others putting together diabetes educational programs.

\section{Strengths and Limitations}

We utilized a pragmatic design in a real world setting that focused on a Mexican American population, therefore, our findings are generalizable to similar populations seeking care in clinics in the US. One of the strengths of our pragmatic design is that we were able to make adaptations as the study was implemented in order to better reflect real world experience. Another strength is our inclusion of a population that suffers significant diabetes-related health disparities that is relatively understudied. Our robust evaluation is also a strength we included both behavioral and objective clinical outcomes ascertained with validated surveys and an objective medical record review. We were also able to provide detailed outcomes stratified by attendance in order to understand the pattern of outcome depending on degree of exposure to the intervention. One of the main strengths of our program is that the health educator was a peer Hispanic female that underwent specific program training and the classes were taught in a group. Consequently the participants felt comfortable discussing issues with the educator and also gained the benefits of a group process for support and accountability. Also, the intervention was performed in a clinical practice setting well known to participants. This education program was supported by funding that allowed adequate infrastructure and personnel to provide the classes for 35 months.

Study findings should be interpreted in the context of study limitations. This study employed a quasi-experimental design with a non-randomized one-group pre-post intervention design. Although we observed an association between the educational intervention and some clinical outcomes, we have to acknowledge that the lack of randomization and absence of a comparison group mean that threats to internal validity exist, for instance we may not have accounted for important 
confounding variables or maturation effects. This study was a pragmatic design reflecting the practices in real-life routine conditions to evaluate the effectiveness of the intervention. Although this study would be unable to estimate the net effect due to an absence of a control group, it provides the effect size of the intervention in real world practical conditions.

As a quasi-experimental design, it is prone to a greater tendency for attrition bias and missing responses. One of the main challenges of a longitudinal program in clinical or community settings is the attrition rate. In this study, the total number of participants that attended $100 \%$ of classes was 66 , an attrition rate of $68 \%$; for completers of $75 \%$ of classes the attrition rate was $57.4 \%$. These attrition rates are higher than the $46 \%$ reported by Ryan et al [37] in a non-randomized 4-week diabetes self-management education program facilitated by a nurse and licensed dietician that included non-Hispanic black and Hispanic participants. Our attrition represents the real world experience. It is therefore important to understand and explore the pattern of response by session completion. In this study, we attempted to do just that by conducting paired statistical tests, and conducting analyses stratified by attendance. In doing so we may have overestimated the overall effect; however, we do provide information about the effectiveness of the program in those that do attend. This is important for real world application, as it may help in deciding about resource allocation by clinics and programs. We observed that as long as people attend at least $50 \%$ of the program sessions, they do gain a benefit, indicating that resources could be diverted to increasing attendance to at least $50 \%$ for as many as possible and less so on those already at $50 \%$ or greater attendance. The selfmanagement behaviors were evaluated by self-report and are therefore open to self-report bias. However, since both baseline and follow-up data are prone to this, the effect should be minimized. Also the EMR systems changed half way through data collection so we had to navigate two separate EMRs, and the data review depends on accuracy of information in the EMR.

In summary, culturally tailored diabetes self-management education programs adapted and delivered in a primary care could be important in alleviating diabetes disparities among Mexican Americans. Promoting self-care in an integrated and cultural competent program may improve glycemic control, self-management behaviors and knowledge and beliefs.

\section{Supplementary Material}

Suppl 1. Survey.

\section{Acknowledgments}

We thank Dr. Silvia Aide Hernandez and Dr. Diana Escobedo who helped with entering the clinical data.

\section{Financial Disclosure}

Funding for the implementation of the DEEP program was provided by TMF Quality Institute.

\section{Conflict of Interest}

None to declare.

\section{Informed Consent}

An informed consent and HIPAA consent were obtained from the patients for the study.

\section{Author Contributions}

SFL: conceptualization, statistical analysis, manuscript development, main writer; MP: assisted with EMR chart review, manuscript editing; AKD: assisted with manuscript editing, analysis plan and interpretation of statistical analysis; GS: manuscript editing; NKS: principal investigator, conceptualization, implementation and manuscript development.

\section{Data Availability}

The data supporting the findings of this study are available from the corresponding author upon reasonable request.

\section{References}

1. Centers for Disease Control and Prevention. National diabetes statistics report, 2017. Atlanta, GA: Centers for Disease Control and Prevention, Us Department of Health and Human Services; 2017. https://www.cdc.gov/diabetes/pdfs/data/statistics/national-diabetes-statistics-report. pdf. Accessed on August 1, 2019. https://dev.diabetes. org/sites/default/files/2019-06/cdc-statistics-report-2017. pdf.

2. Lin J, Thompson TJ, Cheng YJ, Zhuo X, Zhang P, Gregg E, Rolka DB. Projection of the future diabetes burden in the United States through 2060. Popul Health Metr. 2018;16(1):9.

3. Dall TM, Yang W, Halder P, Pang B, Massoudi M, Wintfeld N, Semilla AP, et al. The economic burden of elevated blood glucose levels in 2012: diagnosed and undiagnosed diabetes, gestational diabetes mellitus, and prediabetes. Diabetes Care. 2014;37(12):3172-3179.

4. Alva ML, Hoerger TJ, Zhang P, Cheng YJ. State-level diabetes-attributable mortality and years of life lost in the United States. Ann Epidemiol. 2018;28(11):790-795.

5. The Texas Demographic Center. Diabetes in Texas report 2017. https://demographics.texas.gov/Resources/publications/2018/2018_12_17_DiabetesProfile.pdf. Accessed on August 15, 2019 .

6. American Diabetes Association. Arlington VA. Statistics about diabetes. https://www.diabetes.org/resources/sta- 
tistics/statistics-about-diabetes. Accessed on August 20, 2019.

7. Bayer FJ, Galusha D, Slade M, Chu IM, Taiwo O, Cullen MR. Process of care compliance is associated with fewer diabetes complications. Am J Manag Care. 2014;20(1):41-52.

8. Campbell JA, Walker RJ, Smalls BL, Egede LE. Glucose control in diabetes: the impact of racial differences on monitoring and outcomes. Endocrine. 2012;42(3):471-482.

9. Lee J, Liu C, Sales CE. Racial and ethnic differences in diabetes care and health care use and cost. Prev Chronic Dis. 2006;3:A85.

10. US Department of Health and Human Services Office of Minority Health. Diabetes and Hispanic Americans. https://minorityhealth.hhs.gov/omh/browse. aspx?lvl=4\&lvlid=63. Accessed on August 22, 2019.

11. American Association of Diabetes Educators. AADE7 Self-Care Behaviors. https://www.diabeteseducator.org/ living-with-diabetes/aade7-self-care-behaviors. Accessed on August 28, 2019.

12. Hu J, Wallace DC, McCoy TP, Amirehsani KA. A familybased diabetes intervention for Hispanic adults and their family members. Diabetes Educ. 2014;40(1):48-59.

13. Prezio EA, Cheng D, Balasubramanian BA, Shuval K, Kendzor DE, Culica D. Community Diabetes Education (CoDE) for uninsured Mexican Americans: a randomized controlled trial of a culturally tailored diabetes education and management program led by a community health worker. Diabetes Res Clin Pract. 2013;100(1):19-28.

14. Pena-Purcell NC, Boggess MM, Jimenez N. An empowerment-based diabetes self-management education program for Hispanic/Latinos: a quasi-experimental pilot study. Diabetes Educ. 2011;37(6):770-779.

15. Dauvrin M, Lorant V, d'Hoore W. Is the Chronic Care Model Integrated Into Research Examining Culturally Competent Interventions for Ethnically Diverse Adults With Type 2 Diabetes Mellitus? A Review. Eval Health Prof. 2015;38(4):435-463.

16. Ricci-Cabello I, Ruiz-Perez I, Rojas-Garcia A, Pastor G, Rodriguez-Barranco M, Goncalves DC. Characteristics and effectiveness of diabetes self-management educational programs targeted to racial/ethnic minority groups: a systematic review, meta-analysis and meta-regression. BMC Endocr Disord. 2014;14:60.

17. Barrera M, Jr., Castro FG, Strycker LA, Toobert DJ. Cultural adaptations of behavioral health interventions: a progress report. J Consult Clin Psychol. 2013;81(2):196205.

18. Pottie K, Hadi A, Chen J, Welch V, Hawthorne K. Realist review to understand the efficacy of culturally appropriate diabetes education programmes. Diabet Med. 2013;30(9):1017-1025.

19. Haas L, Maryniuk M, Beck J, Cox CE, Duker P, Edwards L, Fisher E, et al. National standards for diabetes self-management education and support. Diabetes Educ. 2012;38(5):619-629.

20. Chrvala CA, Sherr D, Lipman RD. Diabetes self-management education for adults with type 2 diabetes mellitus: A systematic review of the effect on glycemic control. Pa- tient Educ Couns. 2016;99(6):926-943.

21. Hildebrand JA, Billimek J, Lee JA, Sorkin DH, Olshansky EF, Clancy SL, Evangelista LS. Effect of diabetes self-management education on glycemic control in Latino adults with type 2 diabetes: A systematic review and meta-analysis. Patient Educ Couns. 2020;103(2):266-275.

22. United States Census Bureau. Quick facts Socorro city, Texas; El Paso County, Texas: United States.2013-22017. https://www.census.gov/quickfacts/fact/table/socorrocit ytexas, elpasocountytexas,US/POP815217. Accessed on September 5, 2019.

23. UIC Midwest Latino Health Research, Training and Policy Center. Diabetes Empowerment Education Program DEEPTM. 6th edition. Chicago, IL. http:/www.primerohealth.org/wp-content/uploads/DEEP_ENGLISH_ TMF_2013.pdf. Accessed on September 2, 2019.

24. Marin G, Sabogal F, VanOss Marin B, Otero-Sabogal F, Perez-Stable EJ. Development of a short acculturation scale for Hispanics. Hispanic Journal of Behavioral Sciences. 1987;9:183-205.

25. Garcia AA, Villagomez ET, Brown SA, Kouzekanani K, Hanis CL. The Starr County Diabetes Education Study: development of the Spanish-language diabetes knowledge questionnaire. Diabetes Care. 2001;24(1):16-21.

26. Fernandez S, Olendzki B, Rosal MC. A dietary behaviors measure for use with low-income, Spanish-speaking Caribbean Latinos with type 2 diabetes: the Latino Dietary Behaviors Questionnaire. JAm Diet Assoc. 2011;111(4):589-599.

27. Fisher L, Polonsky WH, Hessler D. Addressing diabetes distress in clinical care: a practical guide. Diabet Med. 2019;36(7):803-812.

28. Egede LE, Ellis C. Development and psychometric properties of the 12-item diabetes fatalism scale. J Gen Intern Med. 2010;25(1):61-66.

29. Hawthorne K, Robles Y, Cannings-John R, Edwards AG. Culturally appropriate health education for Type 2 diabetes in ethnic minority groups: a systematic and narrative review of randomized controlled trials. Diabet Med. 2010;27(6):613-623.

30. Nathan DM, Kuenen J, Borg R, Zheng H, Schoenfeld D, Heine RJ, the Alc-Derived Average Glucose (ADAG) Study Group. Translating the A1C assay into estimated average glucose values. Diabetes Care. 2008;31(8):14731478.

31. Brown SA, Garcia AA, Kouzekanani K, Hanis CL. Culturally competent diabetes self-management education for Mexican Americans: the Starr County border health initiative. Diabetes Care. 2002;25(2):259-268.

32. Peel E, Douglas M, Lawton J. Self monitoring of blood glucose in type 2 diabetes: longitudinal qualitative study of patients' perspectives. BMJ. 2007;335(7618):493.

33. da Silva FAA, Moreira TMM, Menezes LGC. Chronicity, self care, social and family support: how the patient has? Diabetol Metab Syndr. 2015;7(Suppl 1):A202.

34. Peyrot M, Egede LE, Funnell MM, Hsu WC, Ruggiero L, Siminerio LM, Stuckey HL. US ethnic group differences in self-management in the 2nd diabetes attitudes, wishes and needs (DAWN2) study. J Diabetes Complications. 
2018;32(6):586-592.

35. Castillo A, Giachello A, Bates R, Concha J, Ramirez V, Sanchez C, Pinsker E, et al. Community-based diabetes education for Latinos: the diabetes empowerment education program. Diabetes Educ. 2010;36(4):586-594.

36. Song M, Lipman TH. Concept analysis: self-monitoring in type 2 diabetes mellitus. Int J Nurs Stud. 2008;45(11):1700-1710.

37. Ryan JG, Jennings T, Vittoria I, Fedders M. Short and long-term outcomes from a multisession diabetes education program targeting low-income minority patients: a six-month follow up. Clin Ther. 2013;35(1):A43-53.

38. Welch G, Weinger K, Anderson B, Polonsky WH. Responsiveness of the Problem Areas In Diabetes (PAID) questionnaire. Diabet Med. 2003;20(1):69-72.

39. Imazu MF, Faria BN, de Arruda GO, Sales CA, Marcon SS. Effectiveness of individual and group interventions for people with type 2 diabetes. Rev Lat Am Enferma- gem. 2015;23(2):200-207.

40. Burns RJ, Deschenes SS, Schmitz N. Cyclical relationship between depressive symptoms and diabetes distress in people with Type 2 diabetes mellitus: results from the Montreal Evaluation of Diabetes Treatment Cohort Study. Diabet Med. 2015;32(10):1272-1278.

41. Fisher L, Glasgow RE, Strycker LA. The relationship between diabetes distress and clinical depression with glycemic control among patients with type 2 diabetes. Diabetes Care. 2010;33(5):1034-1036.

42. Texas Health and Huma Services. Austin Tx. Texas Behavioral Risk actor Surveillance System (BRFSS) 2017. http://healthdata.dshs.texas.gov/CommunitySurveys/ BRFSS. Accessed on October 10, 2019.

43. Solomon SD, Chew E, Duh EJ, Sobrin L, Sun JK, VanderBeek BL, Wykoff CC, et al. Diabetic retinopathy: a position statement by the American Diabetes Association. Diabetes Care. 2017;40(3):412-418. 